\title{
SURJECTIVE MAPPINGS WHOSE DIFFERENTIAL IS NOWHERE SURJECTIVE
}

\author{
Y. YOMDIN
}

(Communicated by Frederick R. Cohen)

\begin{abstract}
Examples of $C^{k}$-mappings $f: \mathbb{R}^{n} \rightarrow \mathbb{R}^{m}, n \geq m>2$, are given, with rank $d f(x) \leq s$ at any $x \in \mathbb{R}^{n}, 2 \leq s<m$, but $f\left(\mathbb{R}^{n}\right)=\mathbb{R}^{m}$, for any $k<(n-s+2) /(m-s+2)$. Thus a weak form of Sard's theorem (if all the points in the source are critical, the image has measure zero) does not hold for mappings of low smoothness.
\end{abstract}

1. The structure of critical points and values of differentiable mappings has been studied intensively since the classical work of $H$. Whitney [5], [6] and $M$. Morse [3]. Sard's (or, better, M. Morse's-A. P. Morse's-A. Sard's) Theorem [4] claims that critical values of a sufficiently smooth mapping form a set of measure zero. Whitney's paper [5] provides examples of functions which are nonconstant on a connected set of critical points, thus showing the sharpness of the differentiability assumptions in Sard's theorem.

However, the following closely related question seems to be open: Is it possible for a differentiable mapping $f: \mathbb{R}^{n} \rightarrow \mathbb{R}^{m}$, with rank $d f<m$ at every point, to be onto (or to have an image with a nonempty interior)?

Clearly, for $m=1$, any $C^{1}$-function with all the points critical is a constant, so the question makes sense only for mappings into $\mathbb{R}^{m}, m \geq 2$. If Sard's theorem is valid, the answer is, of course, negative. But what happens for mappings of a lower smoothness than that required in Sard's theorem?

Besides its theoretical interest, this question is important in various applications. Recently it has attracted the attention of specialists in control theory [2, p. 59].

In this note, we answer this question positively, constructing highly differentiable surjective mappings $f: \mathbb{R}^{n} \rightarrow \mathbb{R}^{m}$ with rank $d f<m$ everywhere.

2. For $f: \mathbb{R}^{n} \rightarrow \mathbb{R}^{m}$, let us denote as $\sum_{s}(f)$ the set $\left\{x \in \mathbb{R}^{n}\right.$, rank $\left.d f(x) \leq s\right\}$, and let $\Delta_{s}(f)=f\left(\sum_{s}(f)\right)$ be the corresponding set of critical values.

Define the set $\Omega_{s}(f)$ of strongly critical values as follows:

$\Omega_{s}(f)=\left\{y \in \mathbb{R}^{m} / \operatorname{rank} d f(x) \leq s\right.$ for any $\left.x \in f^{-1}(y)\right\}$. While the usual

Received by the editors March 30, 1989.

1980 Mathematics Subject Classification (1985 Revision). Primary 58C25, 58C27.

Supported by NSF Grant DMS-8610730. 
Sard's theorem describes the sets $\Delta(f)$, the question above concerns $\Omega(f)$. We have a diagram of inclusions:

$$
\begin{aligned}
& \Delta_{0}(f) \subseteq \Delta_{1}(f) \subseteq \ldots \subseteq \Delta_{m}(f)=\operatorname{Im} f \\
& \cup \mid \| \\
& \Omega_{0}(f) \subseteq \Omega_{1}(f) \subseteq \ldots \subseteq \Omega_{m}(f)
\end{aligned}
$$

Let $I^{p} \subseteq \mathbb{R}^{p}$ denote a $p$-dimensional unit cube $[0,1]^{p}$. Clearly, it is enough to construct corresponding examples of mappings $f: I^{n} \rightarrow I^{m}$.

Theorem. For any $m \geq 3, n \geq m, 2 \leq s \leq m-1$ and $k<(n-s+2)$ / $(m-s+2)$ there is a $C^{k}$-mapping $f: I^{n} \rightarrow I^{m}$ with $\Omega_{s}(f)=I^{m}$ (i.e., rank $d f \leq s$ everywhere and $\left.f\left(I^{n}\right)=I^{m}\right)$.

Proof. Consider first the case $s=2$. We obtain $f$ as a composition of two mappings $f_{1}: I^{n} \rightarrow I^{2}$ and $f_{2}: I^{2} \rightarrow I^{m}$.

Fix three integers $N_{1}, N_{2}, N_{3}$ such that $N_{1}^{n}=N_{2}^{2}=N_{3}^{m}=N$; e.g., $N_{1}=$ $4^{m}, N_{2}=2^{m n}, N_{3}=4^{n}$. Fix also some $\alpha, 0<\alpha<1$. Subdivide $I^{n}$ (resp. $\left.I^{2}, I^{m}\right)$ into $N$ subcubes $I_{j}^{n}$ (resp. $\left.I_{j}^{2}, I_{j}^{m}\right), j=1, \ldots, N$, of the size $1 / N_{1}$ (resp. $1 / N_{2}, 1 / N_{3}$ ), fixing in each case one of the possible orderings of the corresponding grid.

Inscribe in each $I_{j}^{n}$ (resp. $I_{j}^{2}$ ) a concentric subcube $J_{j}^{n}$ (resp. $J_{j}^{2}$ ) of the size $\alpha / N_{1}$ (resp. $\alpha / N_{2}$ ). Denote by $x_{j}$ (resp. $y_{j}, z_{j}$ ) the lower, lefthand vertices of $J_{j}^{n}$ (resp. $\left.J_{j}^{2}, I_{j}^{m}\right), j=1, \ldots, N$, and let $x_{0}=0 \in \mathbb{R}^{n}$ $\left(y_{0}=0 \in \mathbb{R}^{2}, z_{0}=0 \in \mathbb{R}^{m}\right.$, respectively; see Figure 1).

Now let $\varphi: I^{n} \rightarrow I^{2}$ be a $C^{\infty}$ mapping, such that

1. $\varphi \equiv y_{0}$ on a neighborhood of the boundary $\partial I^{n}$.

2. $\varphi \equiv y_{j}$ on a neighborhood of a subcube $J_{j}^{n}, j=1, \ldots, N$.

3. $\varphi\left(I^{n}\right)$ does not intersect the interiors of $J_{j}^{2}$.

To construct $\varphi$, join the points $y_{0}, y_{1}, \ldots, y_{N}$ by a $C^{\infty}$-smooth curve $L$ in $I^{2}$, which does not intersect the interiors of $J_{j}^{2}$ (Figure 1). Define $\varphi$ to be $y_{0} \in L$ on a neighborhood of $\partial I^{n}$ and to be $y_{j} \in L$ on a neighborhood of each $J_{j}^{n}, j=1, \ldots, N$. Consider $\varphi$ as a partial mapping of $I^{n}$ into $L$ (diffeomorphic to $[0,1]$ ) and extend it to $I^{n}$ in a $C^{\infty}$ manner by Whitney's theorem [6].

Let $\psi: I^{2} \rightarrow I^{m}$ be a $C^{\infty}$-mapping with the following properties:

1. $\varphi \equiv z_{0}$ on a neighborhood of $\partial I^{2}$.

2. $\varphi \equiv z_{j}$ on a neighborhood of each $j_{j}^{2}, j=1, \ldots, N$.

Construction of $\psi$ is immediate.

Now let $f_{1}^{0}=\varphi, f_{2}^{0}=\psi$. To construct $f_{1}^{1}$ (resp. $f_{2}^{1}$ ) replace $\varphi$ (resp. $\psi$ ) on each $J_{j}^{n}$ (resp. $J_{j}^{2}$ ) by an appropriately rescaled copy of $\varphi$ which maps $J_{j}^{n} \rightarrow J_{j}^{2}$ (copy of $\psi$ which maps $J_{j}^{2} \rightarrow I_{j}^{m}$ ). Properties 1 and 2 of $\varphi$ and $\psi$ provide a $C^{\infty}$-smooth contact of the "old" and "new" parts. Applying 


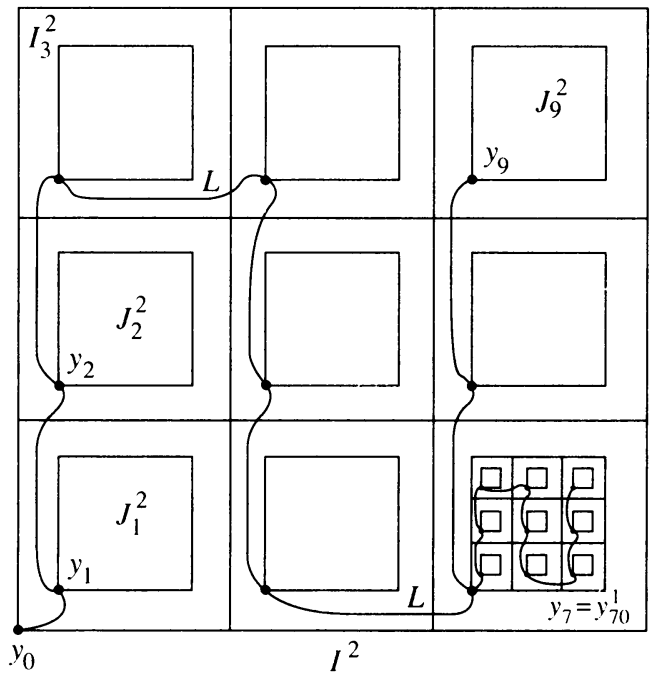

Figure 1

this procedure $r$ times, we obtain $C^{\infty}$-smooth mappings $f_{1}^{r}: I^{n} \rightarrow I^{2}$ and $f_{2}^{r}: I^{2} \rightarrow I^{m}$, and these mappings differ for $r-1$ and $r$ only on the union $\mathscr{D}_{r}^{n} \subset I^{n}\left(\mathscr{D}_{r}^{2} \subset I^{2}\right)$ of the subcubes $J^{n}\left(J^{2}\right)$ of the $r$ th order.

Let $f_{1}=\lim _{r \rightarrow \infty} f_{1}^{r}, f_{2}=\lim _{r \rightarrow \infty} f_{2}^{r}$.

Denote by $G^{n} \subset I^{n}\left(G^{2} \subset I^{2}\right)$ the Cantor sets $\bigcap_{r=1}^{\infty} \mathscr{D}_{r}^{n}\left(\bigcap_{r-1}^{\infty} \mathscr{D}_{r}^{2}\right.$, respectively). Then $G^{2} \subseteq f_{1}\left(G^{n}\right)$ and $f_{2}\left(G^{2}\right)=I^{m}$. This follows by considering the grids $x_{j}^{r}, y_{j}^{r}, z_{j}^{r}$ of all the orders $r$, which are mapped onto one another by $f_{1}$ and $f_{2}$. In particular, $f_{2} \circ f_{1}\left(I^{n}\right)=I^{m}$. By property 3 of $\varphi f_{1}\left(I^{n} \backslash G^{n}\right) \subseteq I^{2} \backslash G^{2}$ and hence $f_{2} \circ f_{1}$ is $C^{\infty}$ on $I^{n} \backslash G^{n}$ (by construction, $f_{1}$ is $C^{\infty}$ on $I^{n} \backslash G^{n}$ and $f_{2}$ is $C^{\infty}$ on $\left.I^{2} \backslash G^{2}\right)$.

It remains to estimate the derivatives of $f_{2} \circ f_{1}(x)$ as $x \in I^{n} \backslash G^{n}$ approaches $G^{n}$.

Once more by property 3 of $\varphi$, for $x \in \mathscr{D}_{r}^{n} \backslash \mathscr{D}_{r+1}^{n} f_{1}(x) \in \mathscr{D}_{r}^{2} \backslash \mathscr{D}_{r+1}^{2}$, for any $r$. Hence computing rescaling factors shows that

$$
\begin{gathered}
f(x)=f_{2} \circ f_{1}(x)=\left[\left(1 / N_{3}\right)^{r} \psi\left(N_{2} / \alpha\right)^{r}\right] \circ\left[\left(\alpha / N_{2}\right)^{r} \varphi\left(N_{1} / \alpha\right)^{r}\right] \\
=(1 / 4)^{n r} \psi \circ \varphi\left(4^{m} / \alpha\right)^{r} .
\end{gathered}
$$

The $k$ th derivative of $f$ satisfies

$$
d^{k} f(x)=(1 / 4)^{n r} d^{k}(\psi \circ \varphi)\left(4^{m} / \alpha\right)^{r k}=\left(4^{m k-n} / \alpha^{k}\right)^{r} d^{k}(\psi \circ \varphi) .
$$

For any $k<n / m_{1}$, this expression tends to zero as $r \rightarrow \infty$ (or $x$ approaches $G^{n}$ ), assuming $\alpha$ to be sufficiently close to 1 . Hence the mapping $f$ constructed is at least $C^{k}$-smooth, with all the derivatives of order $\leq k$ vanishing on $G^{n}$. Since $f$ goes through $\mathbb{R}^{2}$, rank $d f \leq 2$ on $I^{n} \backslash G^{n}$, and it is zero on $G^{n}$. 
This proves the theorem for $s=2$. For $s>2$, consider $I^{n}$ and $I^{m}$ as $I^{n-s+2} \times I^{s-2}$ and $I^{m-s+2} \times I^{s-2}$, respectively, and define $f$ to be as above on the first factor and to be identical on the second.

\section{Open questions.}

1. What is the theorem corresponding to Sard's theorem for the sets $\Omega_{s}$. The remaining differentiability gap is rather small: in the examples above, $f \in C^{k}$, with any $k<(n-s+2) /(m-s+2)$, and for $f \in C^{l}$, $l \geq(n-s) /(m-s)$, the set $\Delta_{s}$, containing $\Omega_{s}$, has measure zero, by the usual Sard's theorem. What is a corresponding result for near-critical values (compare [7])?

2. Is it possible to construct $C^{\infty}$-examples as above on infinite-dimensional spaces? In particular, is it possible for input-to-state mappings of nonlinear control systems? (See [1] for $C^{\infty}$-counterexamples to the usual Sard's theorem in this framework).

3. Our construction excludes $s=1$ (the mean value theorem prevents constructing $\varphi: I^{n} \rightarrow I$ with property 3 above). Are there examples of surjective mappings $f: I^{n} \rightarrow I^{m}$ with rank $d f \leq 1$ everywhere?

\section{ACKNOWLEDGMENT}

The author would like to thank $\mathrm{H}$. Sussmann for asking the question partially answered in this note, and for stimulating discussions.

\section{REFERENCES}

1. M. Briskin and Y. Yomdin, Critical and near-critical values in nonlinear control problems, preprint.

2. W. H. Fleming, Future directions in control theory: a mathematical perspective, Report of the Panel on Future Directions in Control Theory, SIAM Reports, Philadelphia, 1988.

3. M. Morse, The calculus of variations in the large, Amer. Math. Soc. Publ. 18, 1934.

4. A. Sard, The measure of the critical values of differentiable maps, Bull. Amer. Math. Soc. 48 (1942), 883-890.

5. H. Whitney, A function not constant on a connected set of critical point, Duke Math. J. 1 (1935), 514-517.

6. _ Analytic extensions of differentiable functions defined in closed sets, Trans. Amer. Math. Soc. 36 (1934), 63-89.

7. Y. Yomdin, The geometry of critical and near-critical values of differentiable mappings, Math. Ann. 264 (1983), 495-515.

The Weizmann Institute of Science, Rehovot 76100, IsRael 